\title{
Substrate-induced bandgap opening in epitaxial graphene.
}

S. Y. ZHOU, G.-H. GWEON, A. V. FEDOROV, P. N. FIRST, W. A. DE HEER, D.-H. LEE, F. GUINEA, A. H. CASTRO NETO AND A. LANZARA

Nature Materials 6, 770-775 (2007).

The authors wish to make some corrections to the references in the above Article:

1. They wish to add references 20,21 to adjoin reference 9 in the first paragraph regarding single layer and bilayer graphene.

2. They wish to add the following references to adjoin references 7,8 in the first paragraph regarding gaps in graphene ribbons:

Nakada, K., Fujita, M., Dresselhaus, G. \& Dresselhaus, M. S. Edge state in graphene ribbons: nanometer size effect and edge shape dependence. Phys. Rev. B 54, 17954-17961 (1996).

Son, Y. W., Cohen, M. L. \& Louie, S. G. Energy gaps in graphene nanoribbons. Phys. Rev. Lett. 97, 216803 (2006)

Han, M.Y., Ozyilmaz, B., Zhang, Y. \& Kim, P. Energy band-gap engineering of graphene nanoribbons. Phys. Rev. Lett.

98, 206805 (2007).

3. During the long preparation of this manuscript the authors became aware of theoretical work that they wish to add to adjoin references 6-9 in the first paragraph regarding gap opening by breaking carbon sublattice symmetry:

Giovannetti, G., Khomyakov, P. A., Brocks, G., Kelly, P.J. \& van den Brink, J. Substrate-induced band gap in graphene on hexagonal boron nitride: Ab initio density functional calculations. Phys. Rev. B 76, 073103 (2007). 